Sliding motion, robust control and power loss minimization in a class of non-linear switched converters

Olm, J.M.; Biel, D.; Fossas, E.; Zinober, A.S.; Sanz, L.M.

Electronic version of an article published as [International Journal of Control, Volume 80, Issue 6, Year 2007 Pages 968-981] [Article DOI: 10.1080/00207170701261960] (c) [copyright Taylor \& Francis]

[The original publication is available at

http://www.tandf.co.uk/journals/tf/00207179.html] 


\title{
Sliding motion, robust control and power loss minimization in a class of nonlinear switched converters
}

\author{
J. M. OLM $\dagger^{*}$, D. BIEL $\ddagger$ E. FOSSASł, A. S. I. ZINOBER§ and L. M. SANZ§ \\ $\dagger$ Department of Automatic Control and Computer Engineering, Technical University of Catalonia, \\ Avda. Diagonal 647, 08028 Barcelona, Spain \\ $\ddagger$ Institute of Industrial and Control Engineering, Technical University of Catalonia, \\ Av. Diagonal, 647, 08028 Barcelona, Spain \\ $\S$ Department of Applied Mathematics, The University of Sheffield, Sheffield S10 2TN, UK \\ ( $v 1$ released August 2006)
}

\begin{abstract}
In this paper we propose a sliding mode control scheme that allows robust control of the output voltage in a class of nonlinear switched converters that includes the Non-Inverting Buck-Boost, the Watkins-Johnson and the Inverse of Watkins-Johnson. Using a full-state reference profile-based sliding surface, periodic signals may be tracked by the output voltage under appropriate restrictions. Furthermore, semi-infinite programming techniques are used to minimize power loses while taking into account the existence of load perturbations. The procedure consists of choosing a reference signal for the inductor current with minimum root mean square, subject to the maintenance of sliding regime under possible load variation in a set with known bounds. Realistic simulation results show a highly robust performance in the presence of load disturbances.
\end{abstract}

\section{Introduction}

The possibility of using nonlinear DC-DC switching power converters as source inverters has been thoroughly studied during the last twenty years. The main reason for this is that the conventional full-bridge buck converter must incorporate a transformer to adequately perform step-up tasks, thus resulting in a significative increase of the weight and size of the power supply equipment.

When using nonlinear converters, efforts are handicapped by the nonminimum phase character shown by these devices when direct control over the output voltage is exerted (see Fossas et al., 2002, for example). First attempts to solve the problem by means of a current-based indirect control in boost and buck-boost DC-AC inverters (Cáceres et al., 1999; Hernández et al., 2003) have led to systems with sensitivity to external perturbations and parameter uncertainties. On the other hand, direct control strategies of the output voltage that include passivity-based schemes (Rodríguez et al., 2000) and PID-type sliding mode controllers (Tan et al., 2005), have been used for regulation purposes. PI controllers also offer interesting performance in full-bridge nonlinear inverters (Liang et al., 2002; Vázquez et al., 2003; Sanchis et al., 2005a,b). However, it is well known that PI control designs are based on a small signal model; this leads to output waveforms being sensitive to power stage parameter variations, such as the output load.

We address the possibility of using DC-DC converters of the family that includes the Non-Inverting Buck-Boost, the Watkins-Johnson and the Inverse of Watkins-Johnson (Erickson et al., 2001), as source inverters. The main feature of these converters is that they possess two switches. Hence, taking advantage

*Corresponding author. Email: josep.olm@upc.edu

J.M. Olm and E. Fossas are partially supported by CICYT grant DPI2004-06871-C02-02.

D. Biel is partially supported by CICYT grant DPI2006-15627-C03-01.

L.M. Sanz is supported through a European Community Marie Curie CTS Fellowship. Contract number: HPMCT-CT-200100278. 
of the two control inputs, it is possible to design a sliding mode control strategy which is able to yield robust tracking of periodic signals by the output voltage and, at the same time, maintain the input current regulated at a prescribed level. The proposal employs a full-state reference profile-based sliding surface that does not depend on the plant parameters. The converter is assumed to undergo possible load variations in a set with known bounds. Restrictions for candidate signals to be tracked are derived.

The existing literature dealing with the aforementioned converters contains promising results for regulation tasks but, as far as the authors know, none of them has reported control designs for tracking purposes. A synchronous Non-Inverting Buck-Boost converter prototype with a small-signal based controller (Sahu et al., 2004) offers good performance in regulation tasks for low voltage range. In (Gaboriault et al., 2004), the switching logic of the converter allows a smooth transition between buck, boost and buck-boost operation modes, depending on the input and the expected output voltage. Basic performance analysis of Watkins-Johnson and Inverse of Watkins-Johnson converters for regulation purposes is available in (Grant et al., 2003a) and (Grant et al., 2003b), respectively.

A preliminary version of this paper (Fossas et al., 2005) is improved here with the sliding mode control analysis of the robust tracking in a class of nonlinear dynamical systems that includes the family of power converters with two switches. Furthermore, it is already known that proper energy transfer constitutes the main goal of power converters, meaning good efficiency and high output signal quality. Maximizing power efficiency requires minimization of the Root Mean Square (RMS) current flowing in the switching converter. This leads to two different effects, namely, optimization of the losses due to the power switching and minimization of the resistive losses in the inductors. Hence, the design incorporates a procedure to reduce power losses based on semi-infinite programming theory (Hettich et al., 1993) and assuming known upper and lower bounds for the load variation; this technique has been successfully applied to a fullbridge boost converter in (Sanz et al., 2006). Finally, the theoretical development is validated by carrying out illustrative simulations of a Non-Inverting Buck-Boost converter with the realistic power electronics software package PSIM.

The article is organized as follows. Section 2 studies the sliding mode robust tracking control of a class of nonlinear systems that include the above cited family of switching power converters. Section 3 completes the procedure with a proposal to minimize energy loses in such systems. These results are applied to the Non-Inverting Buck-Boost, the Watkins-Johnson and the Inverse of Watkins-Johnson converters in Section 4. An example of the robust tracking of a sinusoidal reference by the output voltage of a NonInverting Buck-Boost system with power loss minimization is presented in Section 5. Section 6 contains the corresponding simulation results, while conclusions and suggestions for further research are presented in Section 7.

\section{Sliding mode robust tracking control of a class of nonlinear systems}

Consider the class of multi-input nonlinear systems with state-space dynamics modelled by

$$
\dot{x}=A x+\delta+[B(x)+C] u,
$$

where $x \in \mathbb{R}^{n}, A, B(x), C$ are $n \times n$ square matrices, $B(x)$ being linear, and $\delta \in \mathbb{R}^{n}$ is a vector. Matrices $A, B(x), C$ and vector $\delta$ depend on a set of characteristic parameters of the system, denoted $\lambda_{1}, \ldots, \lambda_{l}$, which are known to belong to the closed set $\Lambda=\left[\lambda_{1 \text { min }}, \lambda_{1 \text { max }}\right] \times \cdots \times\left[\lambda_{l \text { min }}, \lambda_{\text {lmax }}\right]$. Let also the control input vector $u \in \mathbb{R}^{n}$ be such that its components $u_{1}, \ldots, u_{n}$ have fixed feedback gains, i.e. each $u_{i}$ takes its values in the discrete set $\left\{u_{i}^{-}, u_{i}^{+}\right\}$, and $u_{i}^{-}<u_{i}^{+}$is presumed.

The aim of the paper is to achieve the tracking of a predetermined $T$-periodic reference signal $x_{d}(t)$ by the state vector $x$. Therefore, for the case that $x_{d}$ is $\mathcal{C}^{1}$, the tracking error $e=x-x_{d}$ allows the following description for system (1):

$$
\dot{e}=A e+B(e) u+\left[B\left(x_{d}\right)+C\right] e_{u},
$$


where $e_{u}=u-u_{N}$ and $u_{N}$ is the nominal tracking control:

$$
\dot{x}_{d}=A x_{d}+\delta+\left[B\left(x_{d}\right)+C\right] u_{N} .
$$

The control target $e=0$ will be achieved by means of sliding motion. Let $\sigma(e, t)$ be defined as the linear time-dependent surface

$$
\sigma(e, t)=M(t) e
$$

$M(t)$ being nonsingular. The ideal continuous control $u_{e q}(t)$ that makes $\sigma(e, t)$ invariant by the dynamics defined in (2), also known as the equivalent control, is obtained solving

$$
\sigma(e, t)=0, \quad \dot{\sigma}(e, t)=0 .
$$

Therefore, the nonsingularity of $M(t)$ yields

$$
\sigma(e, t)=0 \Longleftrightarrow e=0,
$$

and also

$$
\left.\dot{\sigma}(e, t)\right|_{e=0}=0 \Longleftrightarrow\left[B\left(x_{d}\right)+C\right]\left(u_{e q}-u_{N}\right)=0 \text { and } e=0,
$$

which entails

$$
u_{e q}(t)=u_{N}=\left[B\left(x_{d}(t)\right)+C\right]^{-1}\left[\dot{x}_{d}(t)-A x_{d}(t)-\delta\right]
$$

for the case $\left[B\left(x_{d}(t)\right)+C\right]$ nonsingular. Moreover, the equivalent control $u_{e q}(t)$ is required to lie within the $\mathbb{R}^{n}$ region $\left(u_{1}^{-}, u_{1}^{+}\right) \times \cdots \times\left(u_{n}^{-}, u_{n}^{+}\right)$in order to prevent undesirable effects of control action saturation in the steady state.

Remark 2.1 Note in (4) that the intersection of the switching manifolds $\sigma_{i}=0$ coincides with that of $e_{i}=0$. So, the tracking error vanishes on the sliding surface (3). Furthermore, if $\|\dot{M}(t)\|$ and $\left\|M^{-1}(t)\right\|$ are bounded, the sliding equations associated with the switching surfaces $\sigma_{i}(e, t)=0$ are identical to those associated with $e_{i}=0$ (Utkin, 1992).

Let us now state the following hypotheses:

H1. $A$ is a negative semidefinite matrix.

H2. $B(x)$ is a linear matrix satisfying $x^{\top} B(x)=0 \in \mathbb{R}^{n}, \forall x \in \mathbb{R}^{n}$.

H3. The command profile $x_{d}(t)$ is $\mathcal{C}^{1}$ and $T$-periodic, and is such that the time-dependent $n \times n$ square matrix $D(t)$ defined below is nonsingular $\forall t \geq 0$ :

$$
D(t)=B\left(x_{d}(t)\right)+C .
$$

H4. The equivalent control (5), which can be written as

$$
u_{e q}(t)=D^{-1}(t)\left[\dot{x}_{d}(t)-A x_{d}(t)-\delta\right],
$$

lies inside the region $\left(u_{1}^{-}, u_{1}^{+}\right) \times \cdots \times\left(u_{n}^{-}, u_{n}^{+}\right) \subset \mathbb{R}^{n}, \forall t \geq 0$.

Remark 2.2 Hypotheses $\mathrm{H} 1$ and $\mathrm{H} 2$ relate to the characterization of this class of systems. $\mathrm{H} 3$ is a necessary and sufficient condition for the existence of the equivalent control (6) mentioned in H4. Finally, H4 ensures the non-saturation of the control action in the steady state. As detailed above, Hypotheses H3 and H4 entail necessary conditions on the candidate reference profiles $x_{d}(t)$ for the control goal to be accomplished. 
Proposition 2.1 Let

$$
\sigma(e, t)=-D^{\top}(t) e
$$

be a switching surface for system (2), and assume that Hypotheses $\mathrm{H}_{1}$ to $\mathrm{H}_{4}$ hold. Then, the control law defined componentwise as

$$
u_{i}=\left\{\begin{array}{lll}
u_{i}^{+} & \text {if } & \sigma_{i}>0 \\
u_{i}^{-} & \text {if } & \sigma_{i}<0
\end{array},\right.
$$

produces a stable sliding regime of (2) on the intersection of the discontinuity surfaces $\sigma_{i}=0$.

Proof Let the real symmetric matrix $W(t)$ be

$$
W(t)=D^{-1}(t)\left[D^{-1}(t)\right]^{\top}
$$

which is well defined because of H3. This hypothesis also ensures the continuous differentiability of

$$
V(\sigma, t)=\frac{1}{2} \sigma^{\top} W(t) \sigma
$$

$W(t)$ is positive definite by construction, which makes $V(\sigma, t)$ positive definite. Furthermore, as the eigenvalues of $W(t)$ are positive real functions of $t$, we get (see Chen, 1984, for example)

$$
0 \leq \kappa_{\min }(t)\|\sigma\|^{2} \leq 2 V(\sigma, t) \leq \kappa_{\max }(t)\|\sigma\|^{2}, \quad \forall t \geq 0
$$

where $\kappa_{\min }(t), \kappa_{\max }(t)$ are the smallest and largest eigenvalues of $W(t)$, respectively. The continuity and $T$-periodicity of such eigenvalues (recall $\mathrm{H} 3$ again) allow us to conclude that they achieve a maximum and a minimum value in $[0, T]$, i.e. there exist real, positive constants $\rho_{m}, \rho_{M}$ fulfilling

$$
\begin{aligned}
& 2 \rho_{m} \leq \min _{t \in[0, T]}\left\{\kappa_{\text {min }}(t)\right\}, \\
& 2 \rho_{M} \geq \max _{t \in[0, T]}\left\{\kappa_{\text {max }}(t)\right\}
\end{aligned}
$$

Therefore, $V(\sigma, t)$ is lower and upper bounded in each sphere $\|\sigma\|=R$ inside a neighbourhood of $\sigma=0$ by positive quantities depending only on $R$, and these lower and upper bounds $h_{R}=\rho_{m} R^{2}, H_{R}=\rho_{M} R^{2}$, respectively, are such that

$$
\lim _{R \rightarrow 0} H_{R}=0, \quad \lim _{R \rightarrow \infty} h_{R}=\infty
$$

In order to evaluate the derivative of $V(\sigma, t)$ along the trajectories of (2) note that

$$
V(\sigma, t)=\frac{1}{2} e^{\top} e
$$

Thus,

$$
\begin{aligned}
\dot{V}(\sigma, t) & =e^{\top} \dot{e}=e^{\top}\left[A e+B(e) u+D(t) e_{u}\right]= \\
& =e^{\top} A e-\sigma^{\top}\left[u-u_{e q}(t)\right]
\end{aligned}
$$

because of H2, H3, (5), (6) and (7). Moreover, due to H3 and H4, every component $u_{i e q}(t)$ of the equivalent control vector is continuous, $T$-periodic and lies inside $\left(u_{i}^{-}, u_{i}^{+}\right)$; therefore, it reaches maximum and 
minimum values $u_{i e q}^{+}$and $u_{i e q}^{-}$therein, respectively. Denote as $\epsilon=\left(\epsilon_{i}\right)$ the vector with components

$$
\epsilon_{i}=\inf \left\{u_{i}^{+}-u_{i e q}^{+},\left|u_{i}^{-}-u_{i e q}^{-}\right|\right\}, \quad i=1, \ldots, n .
$$

Hence, $\mathrm{H} 1$ and the proposed switching logic yield

$$
\dot{V}(\sigma, t) \leq-\sum_{i=1}^{i=n} \epsilon_{i}\left|\sigma_{i}\right| \leq-\alpha\|\sigma\|_{1}, \quad \alpha=\inf _{i \in\{1, \ldots, n\}}\left\{\epsilon_{i}\right\}>0
$$

By norm equivalence in $\mathbb{R}^{n}$, there exists $\bar{\alpha}>0$ such that

$$
\dot{V}(\sigma, t) \leq-\bar{\alpha}\|\sigma\|
$$

Then, stable sliding mode motion along the intersection of the discontinuity surfaces $\left\{\sigma_{1}=0\right\} \cap\left\{\sigma_{2}=0\right\}$ occurs (Utkin, 1992).

Remark 2.3 The requirement of continuous differentiability for the Lyapunov function is essential for the existence of sliding regime in multi-input systems. Chapter 4 of (Utkin, 1992) contains clarifying examples.

Remark 2.4 Note that, from Remark 2.1:

(i) $-D^{\top}(t)$ being nonsingular by Hypothesis $\mathrm{H} 3$, the intersection of the sliding surfaces $\sigma_{i}=0$ coincides with that of $e_{i}=0$, thus yielding the achievement of the tracking target $x(t)=x_{d}(t)$.

(ii) It follows from Hypotheses $\mathrm{H} 3$ and $\mathrm{H} 4$ that $\left\|-\dot{D}^{\top}(t)\right\|$ and $\left\|-\left[D^{\top}(t)\right]^{-1}\right\|$ are bounded. Hence, the sliding equations associated with $\sigma_{i}(e, t)=0$ are identical to those associated with $e_{i}=0$.

The robustness of the system performance in the sliding regime is analyzed straightforwardly. Let system (1) suffer a disturbance in the following way:

$$
\dot{x}=A x+\delta+[B(x)+C] u+p(x, t)
$$

$p(x, t)$ being a vector field that maps vectors from $\mathbb{R}^{n} \times \mathbb{R}$ onto $\mathbb{R}^{n}$. Note that $p(x, t)$ may model parametric perturbations as well as state-independent external disturbances.

Proposition 2.2 If Hypothesis H3 is fulfilled, every perturbation $p(x, t)$ satisfies the matching condition (Sira-Ramírez, 1988).

Remark 2.5 Proposition 2.2 guarantees the robustness of the converter in the sense that the induced sliding regimes exhibit a so-called strong invariance property (Sira-Ramírez, 1988): the ideal sliding dynamics is independent of the perturbation signal. However, the disturbance may affect the fulfillment of Hypothesis $\mathrm{H} 4$, because the equivalent control $u_{e q}(t)$ transforms into

$$
u_{e q}^{p}(t)=D^{-1}(t)\left[\dot{x}_{d}(t)-A x_{d}(t)-\delta-p\left(x_{d}, t\right)\right] .
$$

Hence, $\mathrm{H} 4$ has to be preserved in order to prevent the loss of sliding motion on $\sigma(e, t)=0$.

\section{Energy minimization of the reference profile}

Assume that system (1) can track a certain $T$-periodic reference signal $x_{d}(t)$ such that Hypothesis $\mathrm{H} 4$ is fulfilled. Assume also that $x_{i d}(t), i=2, \ldots, n$, are fixed, while $x_{1 d}(t)$ may be arbitrarily chosen within the restriction imposed by $\mathrm{H} 4$. In order to simplify the control implementation effort, let us consider a 
truncated Fourier series expansion for $x_{1 d}(t)$ :

$$
x_{1 d}(t)=a_{0}+\sum_{k=1}^{r} a_{k} \cos k \omega t+b_{k} \sin k \omega t, \quad \omega=2 \pi T^{-1} .
$$

A reasonable criterion for the election of the coefficients $a_{0}, \ldots, b_{r}$ is the minimization of the energy associated with the signal $x_{1 d}(t)$, which is strongly related to its Root Mean Square (RMS) (see Section $1)$ :

$$
F\left(a_{0}, a_{1}, \ldots, b_{r}\right)=\sqrt{\frac{1}{T} \int_{0}^{T} x_{1 d}^{2}(t) d t}=\sqrt{a_{0} 2+\sum_{k=1}^{k=r} \frac{a_{k}^{2}+b_{k}^{2}}{2}} .
$$

Then, the problem to be solved consists of choosing $a_{0}, a_{1}, \ldots, b_{r}$ in such a way that the value of the function (9) is minimum on the domain defined by the inequalities derived from the fulfillment of Hypothesis H4. Furthermore, recalling that the system parameters $\lambda_{1}, \ldots, \lambda_{l}$ take values in the known set $\Lambda=\left[\lambda_{1 \min }, \lambda_{1 \max }\right] \times \cdots \times\left[\lambda_{\text {lmin }}, \lambda_{\text {lmax }}\right]$, according to Remark 2.5 it turns out that such restrictions are to be fulfilled $\forall(t, \lambda) \in[0, T] \times \Lambda$, where $\lambda=\left(\lambda_{1}, \ldots, \lambda_{l}\right)^{\top}$. This situation is easily adjustable to the semi-infinite optimization problem stated and solved in what follows.

Firstly, let us establish some assumptions:

Assumption A1. Let the scalar functions $\phi_{i}: \mathbb{R}^{p} \times \mathbb{R}^{q} \longrightarrow \mathbb{R}, i=1, \ldots, m$, be continuously differentiable, and let $Y \subset \mathbb{R}^{q}$ be compact.

Assumption A2. The set

$$
Z^{P}=\left\{z \in \mathbb{R}^{p}, \phi_{i}(z, y) \leq 0, \forall y \in Y, i=1, \ldots, m\right\}
$$

is nonempty, i.e. $Z^{P} \neq \emptyset$.

Lemma 3.1 Let Assumption A1 hold, and let also $\psi_{i}: \mathbb{R}^{p} \longrightarrow \mathbb{R}, i=1, \ldots, m$, be defined as follows:

$$
\psi_{i}(z)=\max _{y \in Y}\left\{\phi_{i}(z, y)\right\}, \quad i=1, \ldots, m
$$

Then, $\psi_{i}(z)$ is continuous $\forall i=1, \ldots, m$.

Proof (Adapted from (Polak, 1987)) Note that $\psi_{i}(z), i=1, \ldots, m$, exists $\forall z \in \mathbb{R}^{p}$ because of the continuity of $\phi_{i}$ and the compactness of $Y$, which are guaranteed by Assumption A1. Let us fix $i \in\{1, \ldots, m\}$ and $z_{0} \in \mathbb{R}^{p}$, and let $y_{0} \in Y$ be such that $\psi_{i}\left(z_{0}\right)=\phi_{i}\left(z_{0}, y_{0}\right)$. Consider now the open ball $B\left(z_{0}, L\right)$, centered at $z_{0}$ and with arbitrary radius $L$. Then, $\forall z \in B\left(z_{0}, L\right), \exists y \in Y$ verifying

$$
\begin{aligned}
\psi_{i}(z)-\psi_{i}\left(z_{0}\right) & =\phi_{i}(z, y)-\phi_{i}\left(z_{0}, y_{0}\right)=\left[\phi_{i}(z, y)-\phi_{i}\left(z_{0}, y\right)\right]+\left[\phi_{i}\left(z_{0}, y\right)-\phi_{i}\left(z_{0}, y_{0}\right)\right] \leq \\
& \leq \phi_{i}(z, y)-\phi_{i}\left(z_{0}, y\right),
\end{aligned}
$$

because $\psi_{i}\left(z_{0}\right)=\phi_{i}\left(z_{0}, y_{0}\right) \geq \phi_{i}\left(z_{0}, y\right), Y \ni y \neq y_{0}$. The mean value theorem and the Cauchy-Schwarz inequality ensure the existence of $z_{1} \in B\left(z_{0}, L\right)$ satisfying

$$
\phi_{i}(z, y)-\phi_{i}\left(z_{0}, y\right) \leq\left\|\left[\frac{\partial \phi_{i}(z, y)}{\partial z}\right]_{\left(z_{1}, y\right)}\right\|\left\|z-z_{0}\right\| .
$$


Since $\frac{\partial \phi_{i}(z, y)}{\partial z}$ is continuous and $\operatorname{cl} B\left(z_{0}, L\right) \times Y$ is compact by Assumption A1, $K_{i} \geq 0$ is such that

$$
K_{i} \geq \sup \left\{\left\|\frac{\partial \phi_{i}(z, y)}{\partial z}\right\|, \forall(z, y) \in B\left(z_{0}, L\right) \times Y\right\}
$$

Therefore,

$$
\psi_{i}(z)-\psi_{i}\left(z_{0}\right) \leq K_{i}\left\|z-z_{0}\right\|
$$

Interchanging $z$ and $z_{0}$ in (11) and using (12), we get

$$
\left\|\psi_{i}(z)-\psi_{i}\left(z_{0}\right)\right\| \leq K_{i}\left\|z-z_{0}\right\|, \quad \forall z \in B\left(z_{0}, L\right)
$$

The continuity of $\psi_{i}(\cdot)$ in $z_{0}$ follows straightforwardly and, $z_{0}$ being arbitrary, we obtain that $\psi_{i}(\cdot)$ is continuous in $\mathbb{R}^{p}, \forall i \in\{1, \ldots, m\}$.

Lemma 3.2 Let Assumptions A1 and A2 hold. Then, the set $Z^{P}$ defined in (10) is such that:

(i) $z \in Z^{P}$ iff $\psi_{i}(z) \leq 0, \forall i=1, \ldots, p$.

(ii) $Z^{P}$ is closed.

Proof (i) Trivial.

(ii) $Z^{P}$ may be seen as the intersection of the inverse image of the closed subset $(-\infty, 0]$ by $\psi_{i}(\cdot), i=$ $1, \ldots, m$, which are continuous by Lemma 3.1. Then,

$$
Z^{P}=\psi_{1}^{-1}((-\infty, 0]) \cap \ldots \cap \psi_{m}^{-1}((-\infty, 0])
$$

is closed.

We are now ready to pose and solve the following Problem:

Problem P1. Find $z \in \mathbb{R}^{p}$ that minimizes the real valued function

$$
F(z)=\|z\|
$$

on the domain defined by

$$
Z^{P}=\left\{z \in \mathbb{R}^{p}, \quad \psi_{i}(z) \leq 0, \quad i=1, \ldots, p\right\}
$$

Proposition 3.3 Let Assumptions A1 and A2 hold. Then, Problem P1 has a solution in $Z^{P}$.

Proof $Z^{P}$ is nonempty by Assumption A2. Let $\bar{z} \in Z^{P}$ and consider the closed ball $\bar{B}(0, r) \subset \mathbb{R}^{p}$ centered in $0 \in \mathbb{R}^{p}$ and with radius $r=\|\bar{z}\|$. Therefore, the set $\bar{Z}^{P}=\bar{B}(0, r) \cap Z^{P}$ :

(a) It is nonempty by construction, because it contains at least $\bar{z}$.

(b) If $\mathrm{P} 1$ has a solution, it belongs to $\bar{Z}^{P}$, because

$$
\|z\|>\|\bar{z}\|, \quad \forall z \in Z^{P} \backslash \bar{Z}^{P}, \forall \bar{z} \in \bar{Z}^{P} .
$$

(c) It is compact because $\bar{B}(0, r)$ is compact and $Z^{P}$ is closed by Lemma 3.2. Then, $\bar{Z}^{P}$ is a closed subset of a compact set, thus compact.

Hence, the result follows due to the fact that continuous functions, such as $F(\bar{z})$, exhibit minimum value in compact sets. 
Table 1. State space descriptors of a class of switched power converters

\begin{tabular}{lcc}
\hline Converters & $k_{1}$ & $k_{2}$ \\
\hline Non-Inverting Buck-Boost & 0 & 0 \\
Watkins-Johnson & 1 & 0 \\
Inverse of Watkins-Johnson & 0 & 1 \\
\hline
\end{tabular}

4 Robust output voltage tracking with power loses minimization in basic nonlinear DC-to-DC converters with two switches

The basic nonlinear switched converters Non-Inverting Buck-Boost, Watkins-Johnson and Inverse of Watkins-Johnson (Erickson et al., 2001) have a general state-space representation in terms of a twodimensional system with the inductor current $i_{\mathrm{L}}$ and the capacitor voltage $v_{\mathrm{C}}$ as state variables. For a systematic analysis it is advisable to minimize the number of parameters of the system. This purpose may be achieved with the change of variables:

$$
x_{1}=\frac{1}{V_{\mathrm{g}}} \sqrt{\frac{L}{C}} i_{\mathrm{L}}, \quad x_{2}=\frac{1}{V_{\mathrm{g}}} v_{\mathrm{C}}, \quad t=\frac{1}{\sqrt{L C}} \tau,
$$

and the introduction of

$$
\lambda_{1}=\frac{1}{R} \sqrt{\frac{L}{C}}>0
$$

which make the system dimensionless:

$$
\begin{aligned}
& \dot{x}_{1}=u_{1}-x_{2} u_{2}+k_{1}\left(u_{2}-1\right)+k_{2} x_{2}\left(1-u_{1}\right) \\
& \dot{x}_{2}=-\lambda_{1} x_{2}+x_{1} u_{2}-k_{2} x_{1}\left(1-u_{1}\right) .
\end{aligned}
$$

The values of the parameters $k_{1}$ and $k_{2}$ for the different converters are summarized in Table 1 . The control gains $u_{1}, u_{2}$ take values in the discrete set $\{0,1\}$. Moreover, as the converters are assumed to undergo possible load variations in the bounded set $\left[R_{\min }, R_{\max }\right], \lambda_{1} \in\left[\lambda_{1 \min }, \lambda_{1 \max }\right]$.

Note that the dynamical system (13),(14) matches the general state-space representation (1):

$$
A=\left(\begin{array}{cc}
0 & k_{2} \\
-k_{2} & -\lambda_{1}
\end{array}\right), \quad \delta=\left(\begin{array}{c}
-k_{1} \\
0
\end{array}\right), \quad B(x)=\left(\begin{array}{rr}
-k_{2} x_{2} & -x_{2} \\
k_{2} x_{1} & x_{1}
\end{array}\right), \quad C=\left(\begin{array}{cc}
1 & k_{1} \\
0 & 0
\end{array}\right)
$$

Furthermore, Hypotheses $\mathrm{H} 1$ and $\mathrm{H} 2$ are trivially fulfilled. Hence, the sliding mode control model studied in Section 2 is straightforwardly applicable to the robust tracking control of any reference $x_{d}(t)=\left(x_{1 d}(t), x_{2 d}(t)\right)^{\top}$ that allows the verification of Hypotheses $\mathrm{H} 3$ and H4:

(i) $D(t)$ is

$$
D(t)=\left(\begin{array}{cc}
1-k_{2} x_{2 d}(t) & k_{1}-x_{2 d}(t) \\
k_{2} x_{1 d}(t) & x_{1 d}(t)
\end{array}\right)
$$

H3 requires $x_{1 d}(t), x_{2 d}(t) \in \mathcal{C}^{1}$ and $T$-periodic, with $x_{1 d}(t) \neq 0, \forall t \in[0, T]$.

(ii) According to (6) and (15), H4 is fulfilled $\forall x_{1 d}(t), x_{2 d}(t)$ such that

$$
0<u_{1 e q}\left(t, \lambda_{1}\right)=\frac{x_{1 d} \dot{x}_{1 d}+x_{2 d}\left(\dot{x}_{2 d}+\lambda_{1} x_{2 d}\right)+k_{1}\left[x_{1 d}-\left(\dot{x}_{2 d}+\lambda_{1} x_{2 d}\right)\right]}{x_{1 d}}<1, \quad \forall\left(t, \lambda_{1}\right) \in Y,
$$




$$
0<u_{2 e q}\left(t, \lambda_{1}\right)=\frac{\dot{x}_{2 d}+\lambda_{1} x_{2 d}+k_{2}\left[x_{1 d}\left(1-\dot{x}_{1 d}\right)-x_{2 d}\left(\dot{x}_{2 d}+\lambda_{1} x_{2 d}\right)\right]}{x_{1 d}}<1, \quad \forall\left(t, \lambda_{1}\right) \in Y,
$$

where $Y=[0, T] \times\left[\lambda_{1 \min }, \lambda_{1 \max }\right]$. Note that Remark 2.5 has been taken into account.

Therefore, Proposition 2.1 and Remark 2.4 lead straightforwardly to the following result:

Proposition 4.1 Let $x_{1 d}(t), x_{2 d}(t)$ be $\mathcal{C}^{1}$ and T-periodic, with $x_{1 d}(t) \neq 0, \forall t \in[0, T]$, and such that (16),(17) hold. Let also

$$
\begin{aligned}
& \sigma_{1}(e, t)=-e_{1}+k_{2}\left[x_{2 d}(t) e_{1}-x_{1 d}(t)\right] e_{2} \\
& \sigma_{2}(e, t)=x_{2 d}(t) e_{1}-x_{1 d}(t) e_{2}-k_{1} e_{1}
\end{aligned}
$$

be switching surfaces. Then, the control law

$$
u_{1}=\left\{\begin{array}{lll}
1 & \text { if } & \sigma_{1}>0 \\
0 & \text { if } & \sigma_{1}<0
\end{array}, \quad u_{2}=\left\{\begin{array}{lll}
1 & \text { if } & \sigma_{2}>0 \\
0 & \text { if } & \sigma_{2}<0
\end{array},\right.\right.
$$

yields the tracking target $x_{1}=x_{1 d}(t), x_{2}=x_{2 d}(t)$, in the sliding regime.

The power dissipated in a resistive circuit element is directly proportional to the square of the Root Mean Square (RMS) of the current that flows through the element. Moreover, as the target is the tracking of $T$-periodic output voltage references $x_{2 d}(t)$, it is reasonable to search for inductor reference profiles which are also $T$-periodic. Hence, assuming a truncated Fourier series development for $x_{1 d}$ (see (8)), we may minimize its RMS (9) subjected to the restrictions imposed by H4, i.e. (16) and (17).

Hence, let

$$
x_{1 d}=x_{1 d}(z, t)=z_{1}+\sqrt{2} \sum_{k=1}^{r} z_{2 k} \cos k \omega t+z_{2 k+1} \sin k \omega t, \quad \omega=2 \pi T^{-1}, \quad z \in \mathbb{R}^{2 r+1}, \quad t \in \mathbb{R} .
$$

Let us also denote $y=\left(t, \lambda_{1}\right) \in \mathbb{R}^{2}$ and assign

$$
f(y)=f\left(t, \lambda_{1}\right)=\dot{x}_{2 d}(t)+\lambda_{1} x_{2 d}(t), \text { and } g(y)=g\left(t, \lambda_{1}\right)=x_{2 d}(t)\left[\dot{x}_{2 d}(t)+\lambda_{1} x_{2 d}(t)\right] .
$$

Straightforward calculation allows one to prove the following result:

Proposition 4.2 Let $Y=[0, T] \times\left[\lambda_{1 \text { min }}, \lambda_{1 \text { max }}\right]$ be a compact subset of $\mathbb{R}^{2}$, and consider the scalar functions

$$
\begin{aligned}
& \phi_{1}(z, y)=\phi_{1}\left(z, t, \lambda_{1}\right)=g\left(t, \lambda_{1}\right)+x_{1 d}(z, t)\left[\frac{\partial x_{1 d}(z, t)}{\partial t}-1\right]+k_{1}\left[x_{1 d}(z, t)-f\left(t, \lambda_{1}\right)\right], \\
& \phi_{2}(z, y)=\phi_{2}\left(z, t, \lambda_{1}\right)=-g\left(t, \lambda_{1}\right)-x_{1 d}(z, t) \frac{\partial x_{1 d}(z, t)}{\partial t}-k_{1}\left[x_{1 d}(z, t)-f\left(t, \lambda_{1}\right)\right], \\
& \phi_{3}(z, y)=\phi_{3}\left(z, t, \lambda_{1}\right)=-\left[x_{1 d}(z, t)-f\left(t, \lambda_{1}\right)\right]+k_{2}\left\{x_{1 d}(z, t)\left[1-\frac{\partial x_{1 d}(z, t)}{\partial t}\right]-g\left(t, \lambda_{1}\right)\right\}, \\
& \phi_{4}(z, y)=\phi_{4}\left(z, t, \lambda_{1}\right)=-f\left(t, \lambda_{1}\right)-k_{2}\left\{x_{1 d}(z, t)\left[1-\frac{\partial x_{1 d}(z, t)}{\partial t}\right]-g\left(t, \lambda_{1}\right)\right\} .
\end{aligned}
$$

If $\phi_{i}(z, y)<0, \forall y \in Y, i=1,2,3,4$, then restrictions (16) and (17) are fulfilled.

It is now time to establish restrictions on the output voltage reference profile $x_{2 d}(t)$ in order to ensure the existence of solution for Problem P1. To this end we take into account the following lemma. 
Lemma 4.3 Let $Y=[0, T] \times\left[\lambda_{1 \min }, \lambda_{1 \max }\right]$ be a compact subset of $\mathbb{R}^{2}$. Let also $x_{2 d}(t)$ be a $\mathcal{C}^{1}, T$-periodic function satisfying $x_{2 d}(t) \neq 0$ and $f\left(t, \lambda_{1}\right)=\dot{x}_{2 d}(t)+\lambda_{1} x_{2 d}(t) \neq 0, \forall\left(t, \lambda_{1}\right) \in Y$. Then,

$$
g\left(t, \lambda_{1}\right)=x_{2 d}\left(\dot{x}_{2 d}+\lambda_{1} x_{2 d}\right)>0, \quad \forall\left(t, \lambda_{1}\right) \in Y .
$$

Proof (i) $\dot{x}_{2 d}+\lambda_{1} x_{2 d}>0$ and $x_{2 d}<0$, yields

$$
x_{2 d}(0) e^{-\lambda_{1} t}<x_{2 d}(t)<0, \quad \forall\left(t, \lambda_{1}\right) \in Y,
$$

which cannot be fulfilled by any periodic function because, at instant $t=T$, it should be

$$
x_{2 d}(0) e^{-\lambda_{1} T}<x_{2 d}(T)=x_{2 d}(0)<0 .
$$

(ii) $\dot{x}_{2 d}+\lambda_{1} x_{2 d}<0$ and $x_{2 d}>0$, yields

$$
0<x_{2 d}(t)<x_{2 d}(0) e^{-\lambda_{1} t}, \quad \forall\left(t, \lambda_{1}\right) \in Y,
$$

which is again impossible to fulfil for any periodic function.

Hence, regarding Lemma 4.3, let us now introduce the following hypotheses:

H5. Let $Y=[0, T] \times\left[\lambda_{1 \min }, \lambda_{1 \max }\right]$ be a compact subset of $\mathbb{R}^{2} . x_{2 d}(t)$ is $\mathcal{C}^{2}, T$-periodic and is such that

$$
f\left(t, \lambda_{1}\right)=\dot{x}_{2 d}(t)+\lambda_{1} x_{2 d}(t)>0, \quad \forall\left(t, \lambda_{1}\right) \in Y .
$$

H6.1 $x_{2 d}(t)>0, \forall t \in[0, T]$.

H6.2 $1>x_{2 d}(t)>0, \forall t \in[0, T]$.

H6.3 $x_{2 d}(t)>1, \forall t \in[0, T]$.

The existence of a solution for Problem P1 depends upon the following lemma.

Lemma 4.4 Let $x_{2 d}(t)$ be an output voltage reference. If Hypothesis H5 holds, the functions $\phi_{i}(z, y)$, $i=1,2,3,4$, defined in (19),(20),(21),(22), are continuously differentiable. Moreover, assume that:

(i) $\mathrm{H} 5$ and H6.1 hold for $k_{1}=k_{2}=0$,

(ii) H5 and H6.2 hold for $k_{1}=1, k_{2}=0$,

(iii) $H 5$ and H6.3 hold for $k_{1}=0, k_{2}=1$;

then, the set $Z^{P}$ defined as

$$
Z^{P}=\left\{z \in \mathbb{R}^{2 r+1}, \quad \psi_{i}(z) \leq 0, \quad i=1,2,3,4\right\},
$$

where

$$
\psi_{i}(z)=\max _{y \in Y}\left\{\phi_{i}(z, y)\right\}, \quad i=1,2,3,4
$$

is nonempty.

Proof The continuous differentiability of $\phi_{i}(z, y), i=1,2,3,4$, follows immediately from its own definition and from the fact that $x_{2 d}(t)$ is assumed to be $\mathcal{C}^{2}$ in Hypothesis H5.

It is also straightforward from H5 and any of H6.i, $i=1,2,3$, that $f(y)$ and $g(y)$ are continuous and positive $\forall y \in Y$. Furthermore, $Y$ being compact, $f(y)$ and $g(y)$ reach maximum and minimum values in $Y$, i.e. there exist $f_{M}, f_{m}, g_{M}, g_{m} \in \mathbb{R}^{+}$such that:

$$
f_{M}=\max _{y \in Y}\{f(y)\}, \quad f_{m}=\min _{y \in Y}\{f(y)\}, \quad g_{M}=\max _{y \in Y}\{g(y)\}, \quad g_{m}=\min _{y \in Y}\{g(y)\} .
$$


Let us now see how the hypotheses regarding $x_{2 d}(t)$ require $Z^{P} \neq \emptyset$ in each case:

- $\left(k_{1}=k_{2}=0\right)$ Setting $z_{2}=\cdots=z_{2 r+1}=0$ in (19),(20),(21),(22) one gets:

$$
\begin{array}{ll}
\phi_{1}\left(z_{1}, 0, \ldots, 0 ; y\right)=\phi_{100}\left(z_{1} ; y\right)=g(y)-z_{1}, & \phi_{2}\left(z_{1}, 0, \ldots, 0 ; y\right)=\phi_{200}(y)=-g(y) \\
\phi_{3}\left(z_{1}, 0, \ldots, 0 ; y\right)=\phi_{300}\left(z_{1}, y\right)=f(y)-z_{1}, & \phi_{4}\left(z_{1}, 0, \ldots, 0 ; y\right)=\phi_{400}(y)=-f(y) .
\end{array}
$$

Note that $\phi_{200}(y)<0, \phi_{400}(y)<0, \forall y \in Y$, because of Hypotheses H5 and H6.1.

- $\left(k_{1}=1, k_{2}=0\right)$ Setting $z_{2}=\cdots=z_{2 r+1}=0$ in (19),(20),(21),(22) one gets:

$$
\begin{array}{ll}
\phi_{1}\left(z_{1}, 0, \ldots, 0 ; y\right)=\phi_{110}(y)=g(y)-f(y), & \phi_{2}\left(z_{1}, 0, \ldots, 0 ; y\right)=\phi_{210}\left(z_{1}, y\right)=-g(y)+f(y)-z_{1} \\
\phi_{3}\left(z_{1}, 0, \ldots, 0 ; y\right)=\phi_{310}\left(z_{1}, y\right)=f(y)-z_{1}, & \phi_{4}\left(z_{1}, 0, \ldots, 0 ; y\right)=\phi_{410}(y)=-f(y) .
\end{array}
$$

Note that $\phi_{110}(y)<0, \phi_{410}(y)<0, \forall y \in Y$, because of Hypotheses H5 and H6.2.

- $\left(k_{1}=0, k_{2}=1\right)$ Setting $z_{2}=\cdots=z_{2 r+1}=0$ in (19),(20),(21),(22) one gets:

$$
\begin{array}{ll}
\phi_{1}\left(z_{1}, 0, \ldots, 0 ; y\right)=\phi_{101}\left(z_{1}, y\right)=g(y)-z_{1}, & \phi_{2}\left(z_{1}, 0, \ldots, 0 ; y\right)=\phi_{201}(y)=-g(y) \\
\phi_{3}\left(z_{1}, 0, \ldots, 0 ; y\right)=\phi_{301}(y)=f(y)-g(y), & \phi_{4}\left(z_{1}, 0, \ldots, 0 ; y\right)=\phi_{401}\left(z_{1}, y\right)=-f(y)+g(y)-z_{1} .
\end{array}
$$

Note that $\phi_{201}(y)<0, \phi_{301}(y)<0, \forall y \in Y$, because of Hypotheses H5 and H6.3.

Therefore, the $\mathbb{R}^{2 r+1}$ subset

$$
Z_{1}^{P}=\left\{\left(z_{1}, 0, \ldots, 0\right) \in \mathbb{R}^{2 r+1} ; z_{1} \geq \max \left\{f_{M}, g_{M}\right\}\right\}
$$

is trivially a subset of $Z^{P}$ in each case.

Proposition 4.5 Assume that:

(i) H5 and H6.1 hold for $k_{1}=k_{2}=0$,

(ii) H5 and H6.2 hold $k_{1}=1, k_{2}=0$,

(iii) $H 5$ and H6.3 hold $k_{1}=0, k_{2}=1$.

Then, Problem P1 a has solution in the domain $Z^{P}$ defined in (23).

Proof Proposition 3.3 guarantees the existence of solution of Problem P1 under Assumptions A1 and A2, which follow from the hypotheses established in this Proposition because of Lemma 4.4.

Remark 4.1 Note that the unperturbed situation $\lambda_{1 \min }=\lambda_{1 \max }$ leads to a subset of the domain $Z^{P}$ in which it is also possible to prove the existence of solution for Problem P1 by following Proposition 4.5. (Sanz et al., 2006) contains numerical examples of power loss minimization in a full-bridge boost converter for both the perturbed and unperturbed situations.

\section{Example: robust tracking of a sinusoidal reference with power loses minimization in a Non-Inverting Buck-Boost converter}

According to Section 4, the mathematical model of the Non-Inverting Buck-Boost converter, depicted in Figure 1, is:

$$
\begin{aligned}
& \dot{x}_{1}=u_{1}-x_{2} u_{2} \\
& \dot{x}_{2}=-\lambda_{1} x_{2}+x_{1} u_{2} .
\end{aligned}
$$

Hypotheses H3 and $\mathrm{H} 4$ are fulfilled whenever the current and voltage reference profiles $x_{1 d}(t)$ and $x_{2 d}(t)$ 
are such that $x_{1 d}(t) \neq 0$ and (see (16),(17))

$$
\begin{array}{r}
0<u_{1 e q}\left(t, \lambda_{1}\right)=\frac{x_{1 d} \dot{x}_{1 d}+x_{2 d}\left(\dot{x}_{2 d}+\lambda_{1} x_{2 d}\right)}{x_{1 d}}<1, \quad \forall\left(t, \lambda_{1}\right) \in Y, \\
0<u_{2 e q}\left(t, \lambda_{1}\right)=\frac{\dot{x}_{2 d}+\lambda_{1} x_{2 d}}{x_{1 d}}<1, \quad \forall\left(t, \lambda_{1}\right) \in Y,
\end{array}
$$

where $Y=[0, T] \times\left[\lambda_{1 \min }, \lambda_{1 \max }\right]$. Remark 2.5 has been taken into account.

Following equation (18), the switching surface expression is

$$
\begin{aligned}
& \sigma_{1}(e, t)=-e_{1} \\
& \sigma_{2}(e, t)=x_{2 d}(t) e_{1}-x_{1 d}(t) e_{2},
\end{aligned}
$$

and the control law that yields $x_{1}=x_{1 d}(t), x_{2}=x_{2 d}(t)$, is the one contained in Proposition 4.1. To this end $x_{1 d}(t)$ and $x_{2 d}(t)$ must satisfy the requirements of Proposition 4.1.

The final aim of the study of the device is the possibility of developing a robust inverter. Therefore we exemplify the tracking procedure with a sinusoidal reference. Hence, let us state the following hypothesis: H7. Let $x_{2 d}(t)=A+B \sin \omega t$ be such that

$$
A>B \sqrt{1+\left(\frac{\omega}{\lambda_{1 \min }}\right)^{2}}>0 .
$$

Proposition 5.1 Let $x_{2 d}(t)=A+B \sin \omega t$ be a reference candidate to be tracked by the output voltage of a Non-Inverting Buck-Boost converter. If Hypothesis H7 holds, then Problem P1 has a solution.

Proof Straightforward calculation allows one to see that $x_{2 d}(t)$ satisfies Hypotheses H5 and H6.1. Hence, Proposition 4.5 yields the result.

On the other hand, in the selection of an expression for the inductor current reference, a trade off between effective minimization of the power losses and complexity of the controller structure has to be taken into account. Hence, our proposal is to compare the results associated with constant and first harmonic Fourier expansions for the inductor current reference profile, i.e.

$$
\begin{aligned}
& x_{1 d}(t)=x_{1 d}^{*} \\
& x_{1 d}(t)=a_{0}+a_{1} \cos \omega t+b_{1} \sin \omega t .
\end{aligned}
$$

\subsection{Constant inductor current reference}

Let $x_{2 d}(t)=A+B \sin \omega t$ satisfy H7. Then, it follows from Proposition 5.1 that Problem P1 has a solution for $x_{1 d}=x_{1 d}^{*}$. Moreover, from the proof of Lemma 4.4 a solution is given by

$$
x_{1 d}^{*}=\max \left\{f_{M}, g_{M}\right\},
$$

where $f_{M}$ and $g_{M}$ are now:

$$
\begin{aligned}
f_{M} & =\max _{\left(t, \lambda_{1}\right) \in Y}\left\{\lambda_{1} A+B \omega \cos \omega t+B \lambda_{1} \sin \omega t\right\}, \\
g_{M} & =\max _{\left(t, \lambda_{1}\right) \in Y}\left\{\lambda_{1}\left(A^{2}+\frac{B^{2}}{2}\right)+A B \omega \cos \omega t+2 \lambda_{1} A B \sin \omega t-\frac{\lambda_{1} B^{2}}{2} \cos 2 \omega t+\frac{B^{2} \omega}{2} \sin 2 \omega t\right\} .
\end{aligned}
$$




\subsection{First harmonic Fourier expansion for the inductor current reference}

Proposition 5.1 ensures that Problem P1 has a solution for any sinusoidal voltage reference satisfying H7. Note that in this case it turns out that, following $(22), \phi_{4}(z, y)=\phi_{4}(y)=-f(y)$, and Hypothesis H7 guarantees that $\phi_{4}(y)<0, \forall y \in Y$. Hence, we have to consider only $\phi_{1}, \phi_{2}$ and $\phi_{3}$ in solving Problem P1. Specific expressions for these scalar maps (see $(19),(20),(21))$ are:

$$
\begin{gathered}
\phi_{1}\left(a_{0}, a_{1}, b_{1}, t, \lambda_{1}\right)= \\
\lambda_{1}\left(A^{2}+\frac{B^{2}}{2}\right)-a_{0}-\left[a_{1}-\left(a_{0} b_{1}+A B\right) \omega\right] \cos \omega t-\left(b_{1}+a_{0} a_{1} \omega-2 \lambda_{1} A B\right) \sin \omega t+ \\
-\left(\frac{\lambda_{1} B^{2}}{2}-a_{1} b_{1} \omega\right) \cos 2 \omega t-\left(a_{1}^{2}-b_{1}^{2}-B^{2}\right) \frac{\omega}{2} \sin 2 \omega t \\
\phi_{2}\left(a_{0}, a_{1}, b_{1}, t, \lambda_{1}\right)=-\lambda_{1}\left(A^{2}+\frac{B^{2}}{2}\right)-\left(a_{0} b_{1}+A B\right) \omega \cos \omega t-\left(2 \lambda_{1} A B-a_{0} a_{1} \omega\right) \sin \omega t+ \\
\quad-\left(a_{1} b_{1} \omega-\frac{\lambda_{1} B^{2}}{2}\right) \cos 2 \omega t-\left(B^{2}+b_{1}^{2}-a_{1}^{2}\right) \frac{\omega}{2} \sin 2 \omega t, \\
\phi_{3}\left(a_{0}, a_{1}, b_{1}, t, \lambda_{1}\right)=\lambda_{1} A-a_{0}+\left(B \omega-a_{1}\right) \cos \omega t+\left(B \lambda_{1}-b_{1}\right) \sin \omega t .
\end{gathered}
$$

Once the change of variables

$$
a_{0}=z_{1}, \quad a_{1}=\sqrt{2} z_{2}, \quad b_{1}=\sqrt{2} z_{3}
$$

is introduced in $(27),(28),(29)$, Problem P1 may be numerically solved with

$$
F\left(z_{1}, z_{2}, z_{3}\right)=\sqrt{z_{1}^{2}+z_{2}^{2}+z_{3}^{3}}
$$

by means of the function fseminf, available in the Optimization Toolbox of MATLAB. Essentially, the routine uses cubic and quadratic interpolation techniques to estimate peak values in the semi-infinite constraints. The peak values are used to form a set of constraints that are supplied to a sequential quadratic programming (SQP) method.

\section{Simulation results}

The power electronics software PSIM is used to carry out the simulations. The parameters of the NonInverting Buck-Boost converter are: a DC voltage source $V_{\mathrm{g}}=40 \mathrm{~V}$, a nominal output resistance $R=20 \Omega$, an inductance $L=1 \mathrm{mH}$ with an internal resistance $0.01 \Omega$ and a capacitor $C=60 \mu F$ with an internal resistance $0.01 \Omega$. Each switch is implemented by means of an IGBT with a saturation voltage of $2 V$ and a power diode with a voltage drop of $0.5 \mathrm{~V}$.

Figure 2 shows the complete closed-loop circuitry used to perform the simulations. The sliding mode controllers require the use of multipliers, sums and comparators which can be implemented by means of analogue techniques. A detailed description of the procedure may be found in (Biel et al., 2004). The sliding mode controllers use hysteresis cycle comparators (Bilalović et al., 1983; Bülher, 1986) with hysteresis cycles of $0.02 \mathrm{~V}$ for $\sigma_{1}$ and $0.4 \mathrm{~V}$ for $\sigma_{2}$, as well as zero order holders. Hence, the maximum switching frequency is limited to $120 \mathrm{KHz}$. Expressions of $\sigma_{1}$ and $\sigma_{2}$ are in equation (26).

The output voltage reference for tracking is

$$
v_{\mathrm{Cd}}(\tau)=60+40 \sin 2 \pi \nu \tau
$$


with $\nu=50 H z$. The values of the corresponding normalized variables are:

$$
x_{2 d}(t)=1.5+\sin \omega t,
$$

with $\omega=0.0770$, corresponding to a normalized period of $T=81.65$.

Finally, the converter is assumed to undergo load variations up to $100 \%$ of the nominal value, i.e., from $20 \Omega$ to $40 \Omega$. The load perturbations induce a possible variation of $\lambda_{1}$ in the set $\left[\lambda_{1 \min }, \lambda_{1 \max }\right]=$ $[0.1021,0.2041]$.

These settings guarantee that Hypothesis H7 holds. Hence, Proposition 5.1 ensures that Problem P1 has a solution for both constant and first harmonic Fourier expansion reference profiles for the inductor current. Table 2 contains the optimum values for the reference parameters provided by MATLAB, as well as the corresponding RMS values. Note that the use of a periodic reference for the inductor current entails a RMS reduction of $32.47 \%$ with respect to a constant reference. In terms of power consumption, this grows to $60.84 \%$.

$\begin{aligned} & \text { Table 2. } \\
& \text { reference and associated RSM in normalized variables }\end{aligned}$
\begin{tabular}{lcccc}
$x_{1 d}(t)$ & $a_{0}$ & $a_{1}$ & $b_{1}$ & RMS \\
\hline Constant & 1.2934 & 0 & 0 & 1.2934 \\
Periodic & 0.6891 & 0.1711 & 0.5754 & 0.8093 \\
\hline
\end{tabular}

The fulfillment of restrictions $(24),(25)$, which indicate that the converter is working in the unsaturated zone in the steady-state $\forall\left(t, \lambda_{1}\right) \in Y$, may be visualized by means of a 3D plot of the equivalent controls corresponding to constant and periodic current reference profiles in Figures 3 and 4, respectively. Sectional plots of both figures for $\lambda_{1}=0.2041$, which corresponds to $R=20 \Omega$, are included in Figures 5 and 6 .

When realistic simulations of the converter performance are carried out following the guidelines given in Section 5, the DC terms of the inductor current references provided by MATLAB optimization routines have to be slightly increased for sliding motion to be induced. This is a foreseeable effect of unmodelled dynamics and parasitic resistances. Table 3 provides the real values (in ampères) of the ideal and practical values of the DC components of the inductor current references, as well as the RSM values. The percentage reduction of RSM and power loss when using a periodic rather than a constant inductor current reference are about $35.57 \%$ and $58.49 \%$, respectively. These values are similar to the above reported for the ideal case.

Table 3. Ideal and practical values for the DC term of the inductor current reference and associated RSM

\begin{tabular}{lcccc}
\hline$i_{\mathrm{Ld}}(\tau)$ & $i_{\mathrm{Ld} 0}($ ideal $)$ & $i_{\mathrm{Ld} 0}($ practical $)$ & RMS (ideal) & RMS (practical) \\
\hline Constant & $12.67 \mathrm{~A}$ & $14.00 \mathrm{~A}$ & $12.67 \mathrm{~A}$ & $14.00 \mathrm{~A}$ \\
Periodic & $6.75 \mathrm{~A}$ & $8.00 \mathrm{~A}$ & $7.93 \mathrm{~A}$ & $9.02 \mathrm{~A}$ \\
\hline
\end{tabular}

Simulations of the behaviour of the converter for constant and periodic inductor current references are depicted in Figures 7 and 8, respectively. In both cases, the output voltage performs a precise robust tracking of the reference profile. Meanwhile, the inductor currents also respond as expected and achieve the prescribed references. The load current is included in the plots aiming to show the disturbance forced in the output load parameter, which consists of periodic jumps of $100 \%$ of the nominal value.

\section{Conclusions and suggestions further research}

Robust control of the output voltage in a family of nonlinear switching power converters that includes the Non-Inverting Buck-Boost, the Watkins-Johnson and the Inverse of Watkins-Johnson, is performed by means of sliding modes. The proposed switching surface uses a full state reference profile. Output voltage asymptotic tracking of periodic references under arbitrary load variations in a set with known bounds 
is achieved. Meanwhile, the inductor current is independently regulated, and semi-infinite optimization techniques are used to choose a reference profile that minimizes power losses whilst maintaining the system in the sliding regime. Realistic simulation results for a Non-Inverting Buck-Boost converter validate the proposal.

Further research may study the implementation of the proposed scheme by constructing an experimental prototype. Moreover, issues concerning the behaviour of the proposed scheme under non-resistive loads and the differential connection of two converters in order to have a robust inverter should be addressed.

\section{References}

D. Biel and E. Fossas, "SMC applications in power electronics", in Variable structure systems: from principles to implementation, London, The Institution of Electrical Engineers, 2004.

F. Bilalović, O. Mušić and A. S̆abanović, "Buck Converter Regulator Operating in Sliding Mode", in Proc. of the PCI, 1983, pp. 331-340.

H. Bühler, Réglage par mode de glissement, Presses Polytechniques Romandes, 1986.

R.O. Cáceres and I. Barbi, "A boost DC-AC converter: analysis, design and experimentation", IEEE Trans. Power Electronics, vol. 14, pp. 134-141, January 1999.

C.T. Chen, Linear System Theory and Design, New York: Holt, Rinehart and Winston, 1984.

R. Erickson and D. Maksimović, Fundamentals of Power Electronics, 2nd edition, Norwell, MA: Kluwer Academic Publishers, 2001.

E. Fossas and J.M. Olm, "Asymptotic Tracking in DC-to-DC Nonlinear Power Converters", Discrete and Continuous Dynamical Systems - Series B, vol. 2, pp. 295-307, May 2002.

E. Fossas, J.M. Olm and A.S.I. Zinober, "Sliding Motion and Direct Control of the Output Voltage in a Full Bridge Boost Converter", in Proc. of the 44th IEEE Conference on Decision and Control, 2005, pp. 4760-4765.

M. Gaboriault and A. Notman, "A high efficiency, noninverting, buck-boost DC-DC converter", in Proc. of the 9th Annual IEEE Applied Electronics Conference and Exposition, 2004, pp. 1411-1415.

D.A. Grant and Y. Darroman, "Watkins-Johnson converter completes tapped inductor converter matrix", Electronics Letters, vol. 39, pp. 271-272, February 2003.

D.A. Grant and Y. Darroman, "Inverse Watkins-Johnson converter - analysis reveals its merits", Electronics Letters, vol. 39, pp. 1342-1343, September 2003.

C. Hernández, N. Vázquez, J. Álvarez and J. Arau, "Modified passive-based control law for the boost inverter", in Proc. of the IEEE International Symposium on Industrial Electronics, 2003, vol. 2, pp. 764-768.

R. Hettich and K.O. Kortanek, "Semi-infinite programming: theory, methods and applications", SIAM Review, vol. 35, pp. 380-429, 1993.

T.J. Liang, J.L. Shyu and J.F. Chen, "A novel DC-AC boost inverter", in 37th Intersociety Energy Conversion Engineering Conference, 2002, pp. 629-634.

E. Polak, "On the mathematical foundations of nondifferentiable optimization in engineering design", SIAM Review, vol. 29, pp. 21-89, 1987.

H. Rodríguez, R. Ortega, G. Escobar and N. Baranov, "A robustly stable output feedback saturated controller for the boost DC-to-DC converter", Systems \& Control Letters, vol. 40, pp. 1-8, 2000.

B. Sahu and G.A. Rincón-Mora, "A low voltage, dynamic, noninverting, synchronous buck-boost converter for portable applications", IEEE Trans. on Power Electronics, vol. 19, pp. 443-452, March 2004.

P. Sanchis, A. Ursúa, E. Gubía and L. Marroyo, "Design and experimental operation of a control strategy for the buckboost DCAC inverter", IEE Proc. Electr. Power Appl., vol. 152, pp. 660-668, May 2005.

P. Sanchis, A. Ursúa, E. Gubía and L. Marroyo, "Boost DCAC inverter: a new control strategy", IEEE Transactions on Power Electronics, vol. 20, pp. 343-353, March 2005.

L.M. Sanz, A.S.I. Zinober, D. Biel, E. Fossas and J.M. Olm, "Sliding Control and Optimization in a Full Bridge Boost Converter", in Proc. of the CTS-HYCON Workshop, Paris, July 2006; to be published by Hermes Press (France). 
H. Sira-Ramírez, "Differential Geometric Methods in Variable Structure Control", Int. J. Control, vol. 48, pp. 1359-1390, 1988.

S.C. Tan, Y.M. Lai and C.K. Tse, "Implementation of Pulse-Width-Modulation Based Sliding Mode Controllers for Boost Converters", IEEE Power Electronics Letters, vol. 3, pp. 130-135, December 2005.

V.I. Utkin, "Sliding Modes in Control Optimization". Berlin, Heidelberg: Springer-Verlag, 1992.

N. Vázquez, D. Cortés, C. Hernández, J. Álvarez, J. Arau and Jq. Álvarez, "A new non-linear control strategy for the boost inverter", in Proc. of the 34th Annual IEEE Power Electronics Specialist Conference, 2003, vol. 3, pp. 1403-1407.

E. Zeidler, "Nonlinear functional analysis and its applications - Part IIA: Linear monotone operators". New York: Springer-Verlag, 1990. 


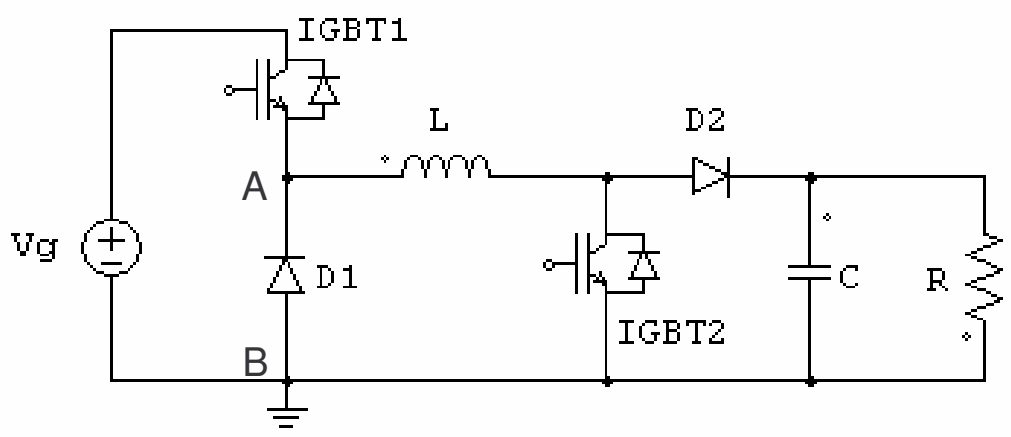

Figure 1. Non-Inverting Buck-Boost converter.

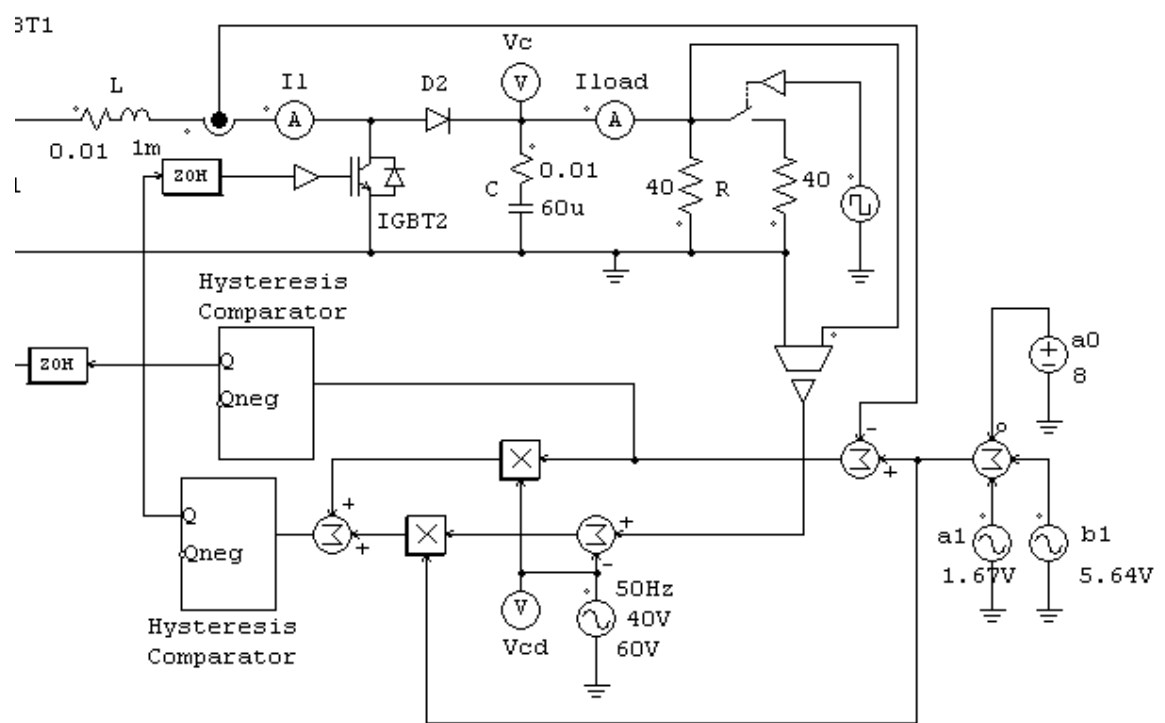

Figure 2. Closed-loop scheme of the Non-Inverting Buck-Boost converter used in the PSIM simulations.

促 


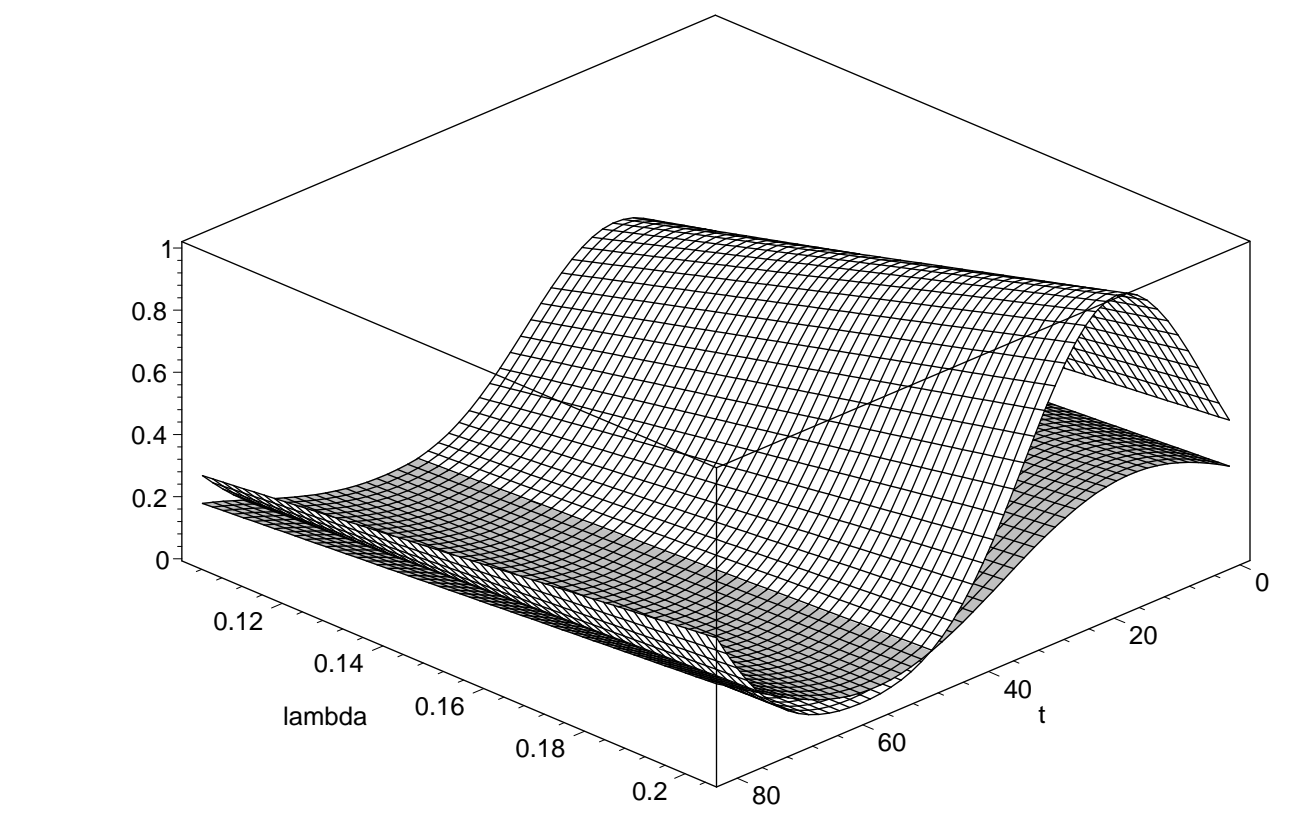

Figure 3. Equivalent control functions $u_{1 e q}\left(t, \lambda_{1}\right)$ (white) and $u_{2 e q}\left(t, \lambda_{1}\right)$ (grey), associated with a constant current reference.

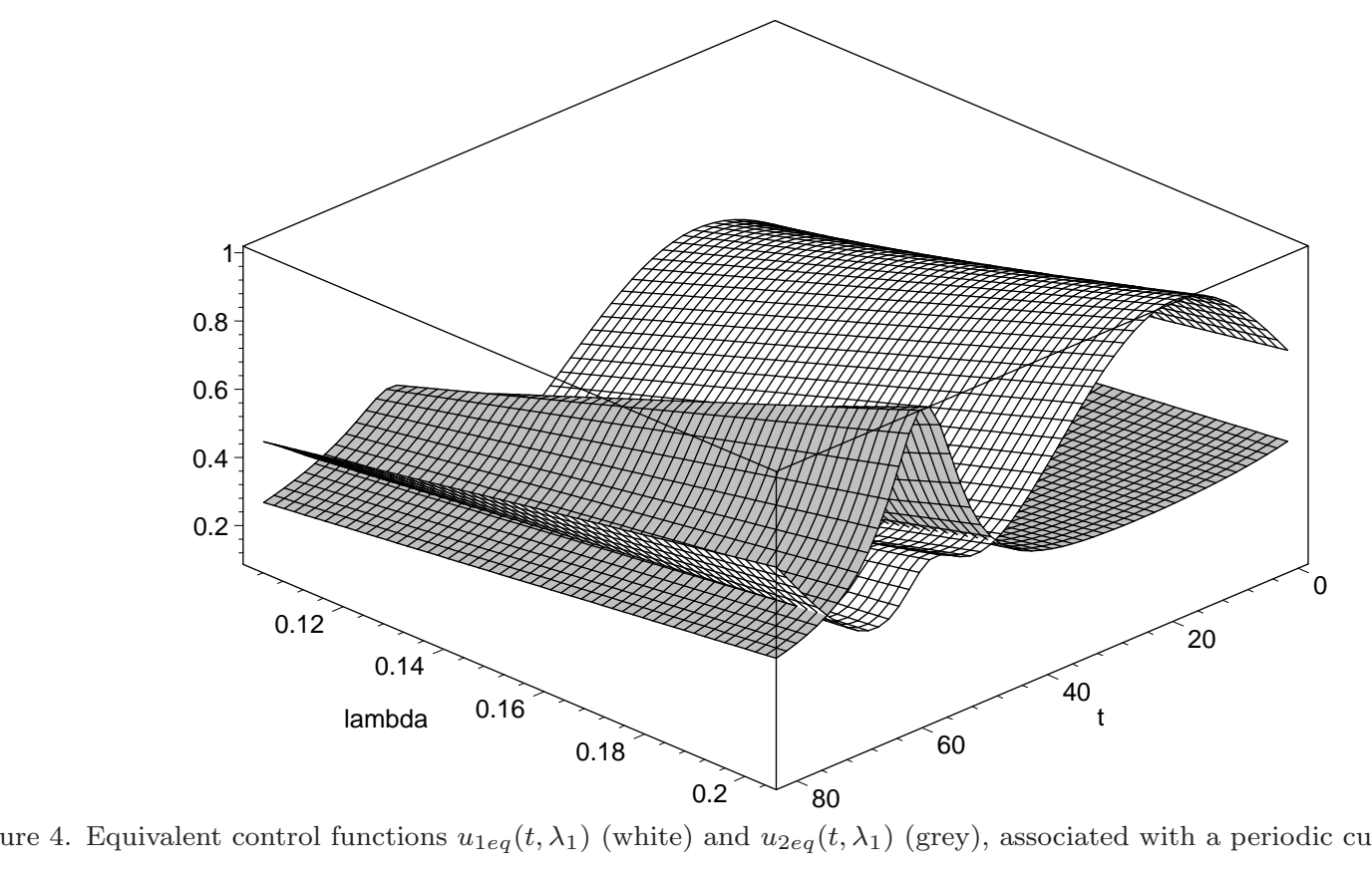

Figure 4. Equivalent control functions $u_{1 e q}\left(t, \lambda_{1}\right)$ (white) and $u_{2 e q}\left(t, \lambda_{1}\right)$ (grey), associated with a periodic current reference.

( 


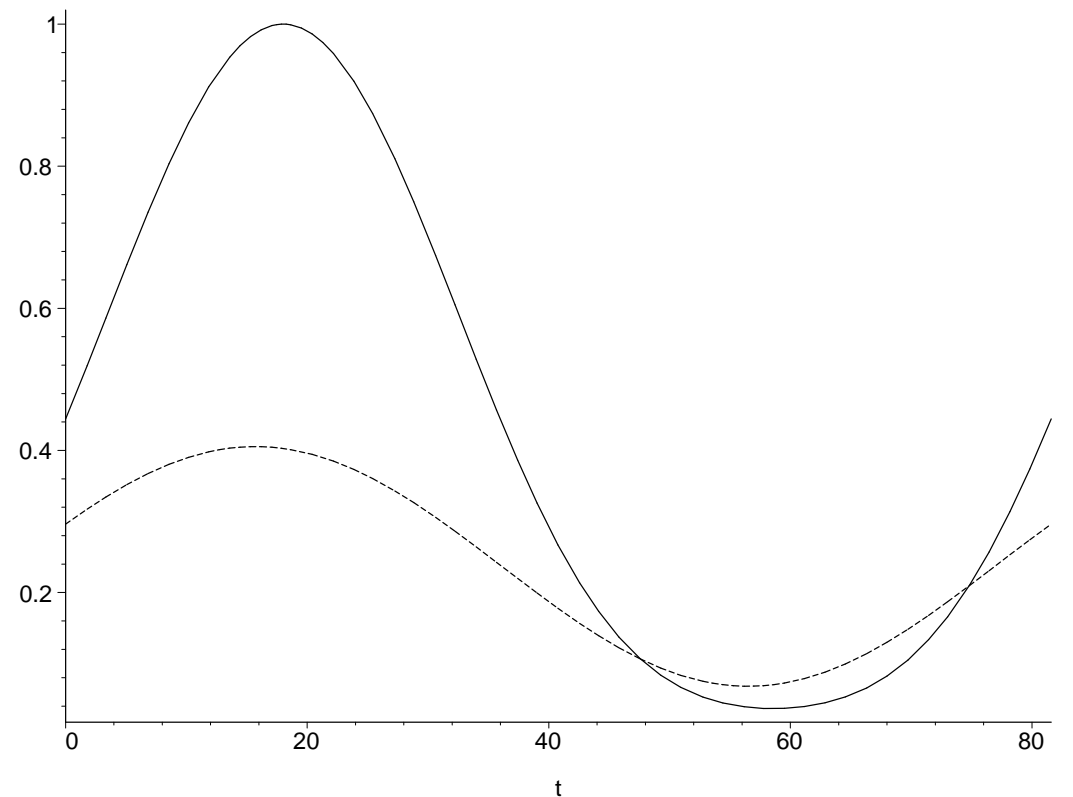

Figure 5. Sectional plot of Figure 2 for $\lambda_{1}=0.2041$. The equivalent controls are $u_{1 e q}\left(t, \lambda_{1}=0.2041\right)$ (solid) and $u_{2 e q}\left(t, \lambda_{1}=0.2041\right)$

(dotted)

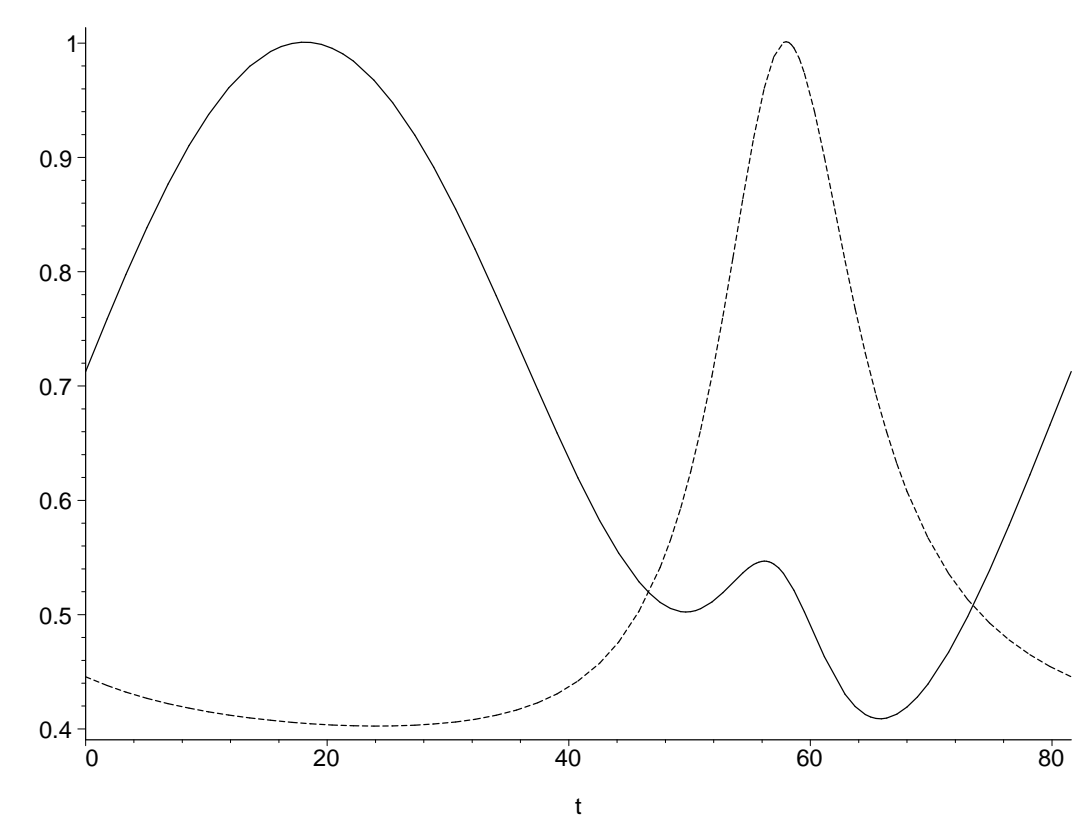

Figure 6. Sectional plot of Figure 3 for $\lambda_{1}=0.2041$. The equivalent controls are $u_{1 e q}\left(t, \lambda_{1}=0.2041\right)$ (solid) and $u_{2 e q}\left(t, \lambda_{1}=0.2041\right)$ (dotted) 


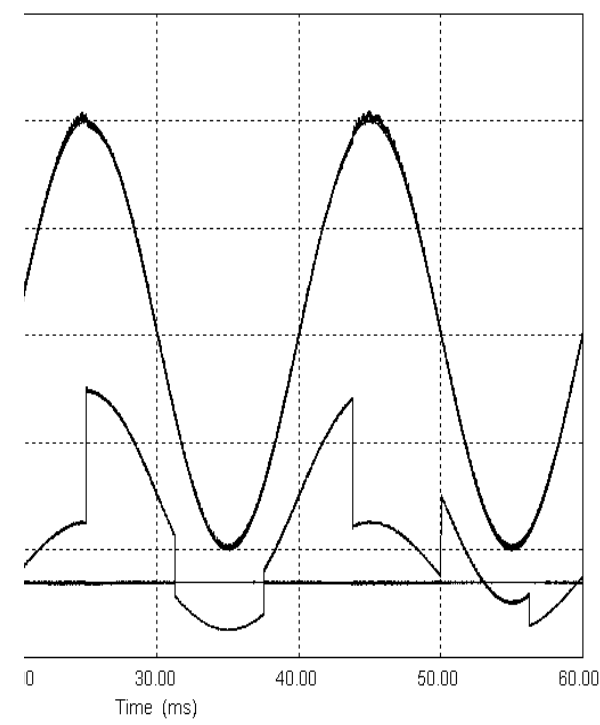

Figure 7. Robust output voltage tracking with a constant inductor current reference: (1) Inductor current $i_{\mathrm{L}},(2)$ Output voltage $v_{\mathrm{C}}$ tracking the reference profile, (3) Load current multiplied by 10.

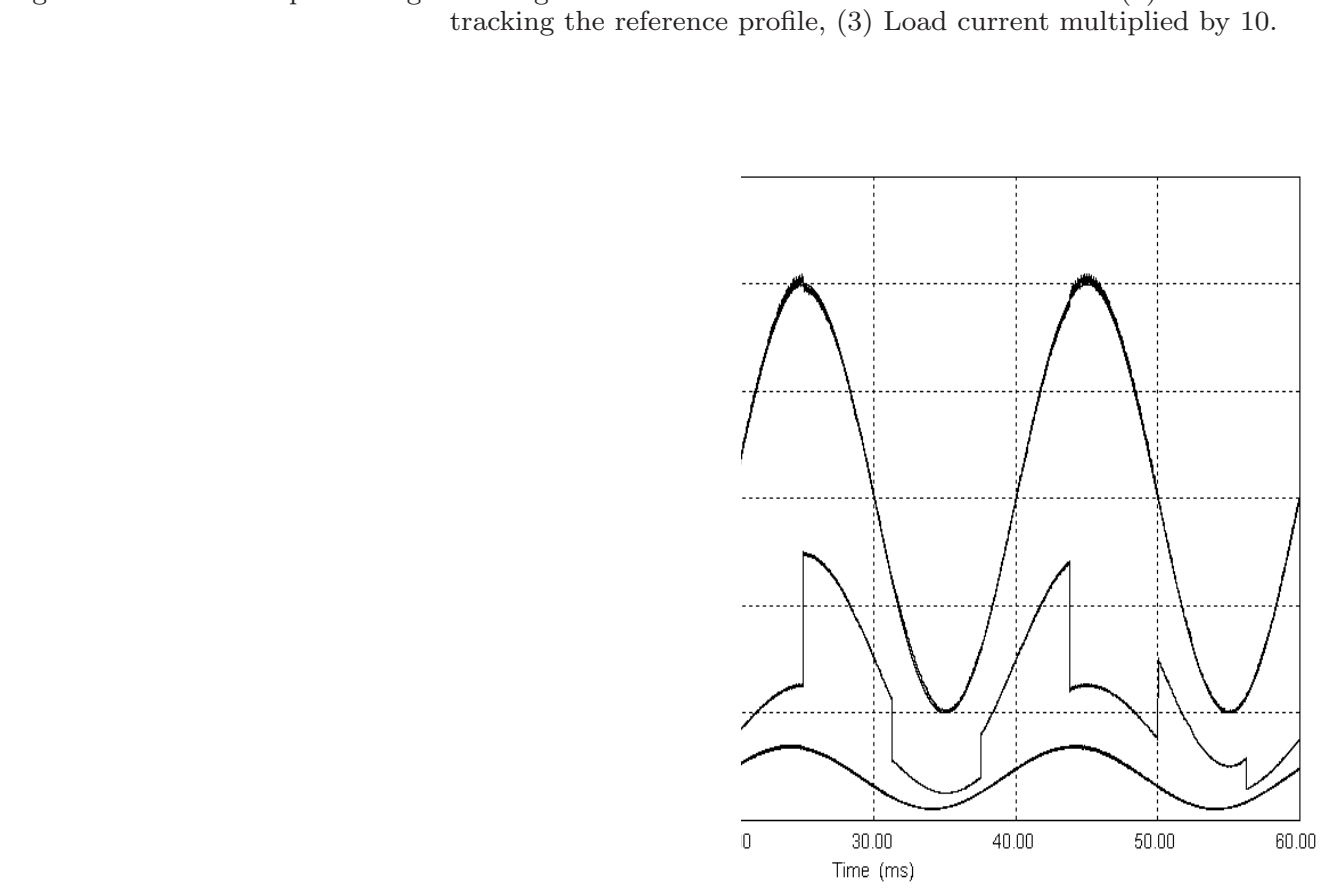

Figure 8. Robust output voltage tracking with a periodic inductor current reference: (1) Inductor current $i_{\mathrm{L}}$, (2) Output voltage $v_{\mathrm{C}}$
tracking the reference profile, (3) Load current multiplied by 10 .

Figure 8. Robust output voltage tracking with a periodic inductor current reference: (1) Inductor current $i_{\mathrm{L}}$, (2) Output voltage $v_{\mathrm{C}}$
tracking the reference profile, (3) Load current multiplied by 10 . 